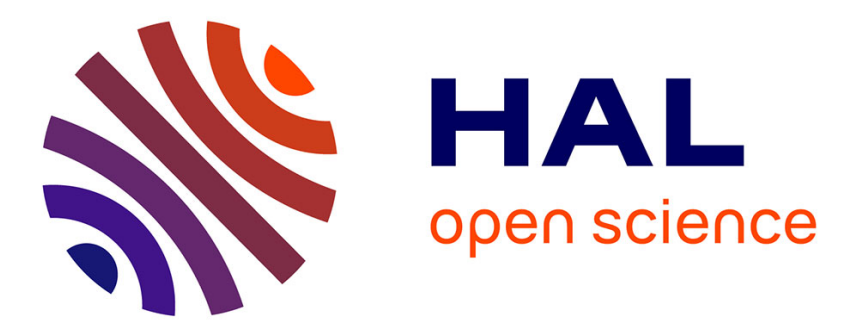

\title{
Ripensare l'esternalizzazione. Per una critica dell'euro-centrismo e dello stato-centrismo negli studi su migrazioni e confini \\ Paolo Cuttitta
}

\section{- To cite this version:}

Paolo Cuttitta. Ripensare l'esternalizzazione. Per una critica dell'euro-centrismo e dello statocentrismo negli studi su migrazioni e confini. Rivista geografica italiana, 2020, 4, pp.55-73. 10.3280/RGI2020-004003 . hal-03242169

\section{HAL Id: hal-03242169 \\ https://sorbonne-paris-nord.hal.science/hal-03242169}

Submitted on 30 May 2021

HAL is a multi-disciplinary open access archive for the deposit and dissemination of scientific research documents, whether they are published or not. The documents may come from teaching and research institutions in France or abroad, or from public or private research centers.
L'archive ouverte pluridisciplinaire HAL, est destinée au dépôt et à la diffusion de documents scientifiques de niveau recherche, publiés ou non, émanant des établissements d'enseignement et de recherche français ou étrangers, des laboratoires publics ou privés. 


\title{
Paolo Cuttitta
}

\section{Ripensare l'esternalizzazione. Per una critica dell'euro-centrismo e dello stato- centrismo negli studi su migrazioni e confini*}

\author{
La versione finale di questo articolo è stata pubblicata in: Rivista Geografica Italiana 127 (4/2020), 55-73
}

Tra le sfide epistemologiche e concettuali con le quali gli studi su migrazioni e confini si sono dovuti confrontare negli scorsi decenni, questo articolo si sofferma in particolare su due, ancora aperte, riguardanti l'esigenza di affrancarsi da due diversi -ismi.

Il primo è il nazionalismo (o statalismo) metodologico (Wimmer e Glick-Schiller, 2002), cioè la tendenza a considerare lo stato come riferimento assoluto della ricerca sociale, e quindi a cadere nella trappola territoriale (Agnew, 1994) dello stato-centrismo. Gli studi sulla governamentalità hanno fornito utili spunti per una critica delle visioni stataliste del potere. Se lo stato è solo un punto - quand'anche il principale - di condensazione dei rapporti di forza (Poulantzas, 1978), il potere - compreso quello di tracciare, difendere, abbattere, ricostruire e trasformare confini; compreso quello di muoversi nello spazio o limitare la libertà altrui di farlo - non può essere ricondotto solo allo stato. L'onnipresenza del potere - e la sua capacità di celarsi dietro il "Potere con iniziale maiuscola" (Raffestin, 1981, p. 63), cioè quello tipicamente incarnato dallo stato, quando in realtà il potere (con la p minuscola) è presente in ogni relazione sociale - corrisponde all'onnipresenza dei confini, e l'onnipresenza dell'uno e degli altri corrisponde all'inafferrabilità della loro identità. Potere e confini, insomma, non sono riconducibili unicamente e semplicemente allo stato, anche quando lo usano per manifestarsi o per nascondervisi dietro. Questo implica l'esigenza di interrogarsi anche sulle dinamiche che determinano le trasformazioni dei confini degli stati e delle loro modalità operative, e guardare alla molteplicità di soggetti che contribuiscono a determinare tali dinamiche e trasformazioni.

Il secondo -ismo sul quale questo articolo si sofferma è un certo nord-centrismo (o settentrionalismo metodologico), ovvero la tendenza della ricerca ad assumere i (più ricchi) paesi del 'Nord globale' come riferimento assoluto. Questa tendenza può articolarsi anche su scala regionale, per esempio manifestandosi in forma di euro-centrismo (o europeismo metodologico), quando il riferimento è l'Europa o uno dei suoi elementi costitutivi - le riflessioni critiche sul nord-centrismo, in effetti, sono in larga parte riflessioni sull'euro-centrismo (Gaibazzi et al., 2017; Genç et al., 2018; Garelli e Tazzioli, 2013; Tazzioli, 2015; Walters, 2015). Anche negli studi di contesti extraeuropei (singoli paesi o intere regioni) c'è una prevalenza di approcci euro-centrici, che insistono nel porre l'Europa come polo principale - la guida e il magnete - della relazione e nell'analizzare ciò che sta fuori dall'Europa solo in rapporto a essa, senza riuscire a decentrare lo sguardo per cogliere la plurivocità, la multidirezionalità e la complessità delle dinamiche in atto. Ciò è particolarmente visibile nel campo degli studi sull'esternalizzazione (Reslow, 2019). Proprio sull'esternalizzazione dei controlli delle migrazioni internazionali questo articolo si concentra nel riflettere criticamente sui limiti derivanti da stato-centrismo ed euro-centrismo.

Il termine 'esternalizzazione' indica il processo con il quale i paesi considerati di destinazione delle migrazioni tentano di esportare determinate politiche di gestione e controllo delle migrazioni nei paesi che sono invece considerati di origine o di 'transito'. Più in generale tale processo di 'esportazione' coinvolge l'insieme di valori, narrative, percezioni, concetti, norme e pratiche associabili al fenomeno migratorio e al desiderio di limitarlo, controllarlo e gestirlo secondo determinati criteri.

Sono trascorsi due decenni da quando la ricerca su migrazioni e confini ha cominciato a occuparsi di esternalizzazione (Andreas, 2003; Lahav e Guiraudon, 2000; Sciortino, 2000; Zolberg, 2003). In questo periodo la produzione scientifica sul tema è cresciuta vertiginosamente, e il concetto di esternalizzazione è diventato un vero e proprio paradigma dei migration and border studies (Bialasiewicz, 2012; Carrera et al., 2019; Cobarrubias et al., 2015; Zaiotti, 2016). Il suo impiego ha infatti consentito di mettere in luce e di analizzare dinamiche importanti su almeno due livelli: il primo è il livello territoriale, che riguarda i luoghi in cui l'azione esternalizzata si dispiega e produce $\mathrm{i}$ propri effetti, così come i nuovi spazi che tale azione produce; il secondo è il livello degli attori, che riguarda i

\footnotetext{
* Il presente lavoro ha beneficiato di un finanziamento del programma dell'Unione Europea per la ricerca e l'innovazione 'Horizon 2020' (accordo di sovvenzione Marie Skłodowska-Curie n. 846320).
} 
soggetti coinvolti, in diversi ruoli e con diverse modalità di partecipazione, nelle attività nelle quali l'esternalizzazione si concretizza.

Nella misura in cui il concetto di esternalizzazione propone l'immagine di un processo di matrice eminentemente statale (si tratta di stati che esternalizzano verso altri stati le funzioni dei propri confini) e unidirezionale (dagli stati di destinazione - segnatamente quelli del 'Nord globale' - a quelli di origine e 'transito'), studiarlo senza cadere nelle trappole nord-centriche e stato-centriche appare particolarmente problematico.

La prima sezione dell'articolo esamina il rapporto tra esternalizzazione e nord-centrismo (nella sua declinazione di euro-centrismo), ${ }^{1}$ mentre la seconda analizza quello tra esternalizzazione e stato-centrismo. Una terza sezione passa in breve rassegna alcuni concetti e approcci metodologici utili a limitare queste distorsioni prospettiche e a meglio inquadrare il concetto di esternalizzazione.

\section{EURO-CENTRISMO}

Una parte consistente della ricerca sulle dinamiche migratorie nei paesi del vicinato allargato dell'UE (dalla Turchia alla Libia, dal Mali all'Egitto etc.) parte dalla prospettiva dell'esternalizzazione, cioè si interroga su se, come e con quali conseguenze l'Europa riesca a esternalizzare i propri confini in tali paesi. Ciò è conseguenza del fatto che i paesi in questione sono considerati tout court come paesi di 'transito'. Düvell (2012), nella sua critica del concetto di 'transito', ha ben spiegato come tale termine sia abusato e applicato indiscriminatamente, e come ciò si debba al fatto che l'etichetta di 'paese di transito' consente di sostenere, da parte degli attori politici dei paesi di destinazione, determinate politiche migratorie, a dispetto del fatto che il dato migratorio nei presunti paesi di ‘transito' è assai più complesso e variegato. Lo stesso euro-centrismo che tende a vedere il ‘transito' ovunque anche dove non c'è - può non solo alimentare le politiche di esternalizzazione ma anche condizionarne lo studio, facendo sì che la prospettiva dell'esternalizzazione prevalga a dispetto della complessità delle dinamiche in campo, fino a individuare l'esternalizzazione anche dove non c'è.

Naturalmente l'esternalizzazione va vista nel contesto più ampio delle politiche internazionali - che si prenda il caso della UE (per esempio la sua politica europea di vicinato) o quello di singoli stati. Nelle relazioni internazionali ogni soggetto mira normalmente, almeno in prima battuta, a soddisfare i propri immediati interessi. È un approccio egocentrico, ed egocentrica è infatti sempre stata la politica europea di vicinato (Kramsch, 2011; Van Houtum e Boedeltje, 2011). Anche l'esternalizzazione dei controlli delle frontiere, dunque, come la politica di vicinato in generale, risponde a una visione egocentrica, la quale, a sua volta, si traduce in una rappresentazione dello spazio di tipo concentrico (Casas-Cortés e Cobarrubias 2019; İşleyen 2018). Di conseguenza, studiare l'esternalizzazione europea significa, necessariamente, anche assumerne in qualche misura la stessa prospettiva euro-centrica e concentrica. È facile finire risucchiati da tali prospettive, lasciando fuori dal campo visivo elementi essenziali per la comprensione dei fenomeni osservati. La ricerca si deve perciò sforzare di relativizzare l'esternalizzazione, inquadrandola in un contesto più ampio, che tenga conto, per ciascuno degli attori in campo, delle diverse dinamiche interne ed esterne, autonome ed eteronome, che ne orientano l'azione.

Il primo accorgimento è - restando ancorati al piano dell'azione governativa, cioè degli attori statali o soprastatali, e restando all'esempio delle politiche europee di vicinato - tenere presente che esiste anche, e va considerata, la agency degli 'europeizzati' (Celata e Coletti, 2016). Qualsiasi *-centrismo si regge sull'assunto che ci sia una periferia, che ci siano dei margini, verso i quali si irradia il potere del centro, verso i quali il centro esercita la propria influenza. Le dinamiche tra centri e margini, però, difficilmente sono univoche, lisce e uniformi (Parker, 2008). Anche in condizioni di squilibrio, esse tendono piuttosto a essere co-determinate dai diversi poli della relazione, i quali contribuiscono, ciascuno secondo i propri mezzi e interessi, e cercando di massimizzare il proprio potere contrattuale, al raggiungimento di un determinato equilibrio o alla sua messa in discussione. I paesi di origine e 'transito' non sono soggetti passivi che si limitano a recepire le politiche e le pratiche esternalizzate dai paesi del 'Nord globale' (Cassarino, 2005; Reslow, 2012), e queste politiche e pratiche non sempre piombano in un vacuum che aspetta solo di essere riempito dall'esterno.

Innanzitutto gli 'esternalizzandi' possono volgere il contesto a proprio vantaggio, sfruttando il potere di negoziazione che le richieste dei secondi inevitabilmente concedono loro. Lo dimostrano la Libia di Gheddafi (Paoletti, 2010) e la Turchia di Erdogan (Beaumont e Smith, 2019). Ciò ricorda che i rapporti di forza possono essere sbilanciati, ma che l'equilibrio è sempre instabile; ci restituisce, quindi, la complessità dei rapporti tra i vari soggetti in campo - rapporti altrimenti interpretabili in modo semplicistico e univoco. Naturalmente ciò non smentisce il paradigma dell'esternalizzazione, anzi: lo conferma. Da un lato, infatti, c'è una richiesta di esternalizzazione; dall'altro la richiesta viene soddisfatta, benché a determinate condizioni. Non dissimile è la

\footnotetext{
${ }^{1}$ L'attività di ricerca di chi scrive si è concentrata, nel corso di diversi anni, principalmente sull'Europa, sui suoi confini e sul suo vicinato, con particolare attenzione al Nordafrica. Gli esempi proposti sono quindi relativi alla regione Mediterranea. Tuttavia le stesse riflessioni potrebbero essere replicate, nella loro sostanza, anche con riferimento ad altri contesti geografici.
} 
situazione in cui la richiesta di esternalizzazione venga respinta (nel suo complesso o, più spesso, in un suo determinato aspetto). Si pensi all'opposizione dei paesi nordafricani all'idea, discussa in ambito UE all'inizio del 2019, di creare piattaforme regionali di sbarco sulla sponda sud del Mediterraneo, o all'atteggiamento con il quale paesi come l'Egitto e la Tunisia continuano a rifiutare o rimandare all'infinito l'approvazione di una legge nazionale sull'asilo, proprio per evitare di diventare i campi profughi dell'Europa. Il fatto che i governi dei paesi in questione rifiutino l'esternalizzazione o un suo aspetto specifico, ovvero ne vincolino l'accettazione a determinate condizioni, o addirittura usino l'esternalizzazione a proprio vantaggio, conferma che l'esternalizzazione dei controlli migratori e delle frontiere è diventato un tema centrale nelle relazioni tra l'Europa e il suo vicinato allargato, ma ricorda che l'esternalizzazione non è un processo semplice e lineare (Bartels, 2018; Collyer, 2016).

Ancora più importante è tenere presente che i regimi migratori vigenti nei paesi di origine e 'transito' possono essere anche, se non soprattutto, il frutto di decisioni prese in modo autonomo dai relativi governi, rispondendo a interessi nazionali o a esigenze locali o regionali, più che a sollecitazioni dei più potenti paesi di destinazione (Cassarino, 2018; El Qadim, 2015; Genç et al., 2018). Addirittura, alcuni paesi - per esempio la Libia di Gheddafi (Pliez, 2004) - hanno adottato misure o posto in essere pratiche restrittive dei movimenti migratori, facendo uso di strumenti come la detenzione amministrativa o l'espulsione, già da prima che il tema del controllo delle migrazioni diventasse centrale nei rapporti con l'Europa. In ogni caso, se è vero che l'esternalizzazione europea ha prodotto effetti tangibili e spesso sconvolgenti (Stock, 2019; Trauner e Deimel, 2013), anche mettendo in crisi economie regionali basate sulle dinamiche transfrontaliere (compresi i movimenti migratori circolari regionali, e non solo - o non tanto - quelli di transito verso l'Europa) (Brachet, 2018; Bredeloup e Pliez, 2005; Pliez, 2006), è anche vero che certe politiche perseguite da determinati paesi possono essere ricondotte a fattori non connessi all'Europa e ai suo sforzi di esternalizzare i propri confini. Lo stesso principio di libera circolazione che l'ECOWAS aveva fatto proprio, e la cui implementazione l'esternalizzazione europea ha certamente contribuito a frenare (Bisong, 2019; Brachet, 2018), non è mai stato effettivamente realizzato anche, se non soprattutto, per ragioni non riconducibili all'esternalizzazione (Bisong, 2019; Okunade e Ogunnubi, 2018).

Un esempio tra i tanti paesi considerati obiettivi dell'esternalizzazione europea è l'Egitto di Al Sisi, che nel 2013 avvia una decisa campagna di discriminazione nei confronti dei numerosi rifugiati siriani. Questi sono dipinti come amici dei Fratelli Musulmani e del deposto presidente Morsi, e quindi nemici del nuovo regime. Ciò produce risultati opposti rispetto a quelli desiderati dall'esternalizzazione, perché il conseguente, sensibile peggioramento delle condizioni di vita per i rifugiati siriani spinge tanti di loro a lasciare l'Egitto per cercare di raggiungere l'Europa. Le partenze di siriani che si imbarcano direttamente dall'Egitto verso l'Italia cominciano, infatti, proprio nell'estate del 2013 (Denaro, 2016). In questo caso si tratta di una politica autonoma che va chiaramente contro gli obiettivi dell'esternalizzazione ma che appare sostanzialmente indipendente dai rapporti tra Egitto ed Europa, e non può dunque essere letta come una forma di opposizione all'esternalizzazione. Naturalmente, poi, le traversate dall'Egitto all'Italia finiscono anche per fare guadagnare all'Egitto potere contrattuale nei rapporti con l'Europa, e l'Egitto arriverà a impedire le partenze dalle proprie coste, tre anni e mezzo dopo l'inizio del fenomeno, quando l'emergenza siriani - dal punto di vista di Al Sisi - sarà ormai ridotta (tra gli ingressi limitati al minimo dall'obbligo del visto e le tante partenze per l'Europa già avvenute) e le contropartite offerte dagli interlocutori europei saranno giudicate soddisfacenti.

La prospettiva euro-centrica può risultare fuorviante anche in altre circostanze, per esempio quando determinate politiche degli "europeizzandi" che risultano funzionali agli obiettivi europei di contenimento delle migrazioni vengono lette come frutto dell'esternalizzazione. Ancora la politica anti-siriana di Al Sisi serve da esempio: l'imposizione dell'obbligo del visto nei confronti dei siriani fu in assoluto una delle prime mosse del presidente egiziano appena preso il potere, ma non può certo leggersi come concessione alle pressioni esternalizzanti europee, perché contestualmente l'Egitto lasciava partire per l'Europa i siriani già presenti nel proprio territorio. L'imposizione del visto può leggersi piuttosto come un'autonoma iniziativa di esternalizzazione egiziana in territorio siriano. Un altro esempio sono i regimi di detenzione e di deportazione che esistono in certi paesi, e che possono esistere anche da prima che l'Europa avviasse le proprie politiche di esternalizzazione. Quando, in un centro di detenzione egiziano, le persone sono spinte al rimpatrio da operatori umanitari che dovrebbero limitarsi a fornire loro cibo o assistenza sanitaria (Cuttitta, 2020), questo può avvenire per dinamiche esclusivamente o prevalentemente interne: per esempio, perché le organizzazioni non governative (ONG), in Egitto, sono sottoposte a pesanti limitazioni e censure di regime, e finiscono per allinearsi agli obiettivi governativi (in questo caso: liberarsi del peso dei migranti indesiderati) anche contro gli interessi dei loro beneficiari. In tal caso ciò che vediamo non sono tanto gli effetti delle politiche europee di esternalizzazione quanto piuttosto gli effetti delle politiche egiziane di internalizzazione dei propri confini, cioè di trasferimento delle funzioni del confine all'interno del territorio statale, poste in essere autonomamente dagli 'esternalizzandi'. I confini - è stato notato ormai da tempo - sono diventati potenzialmente ubiqui (Balibar, 2009), e i processi di 
esternalizzazione vanno di pari passo con quelli di internalizzazione (per esempio i controlli mirati e sistematici in determinate aree urbane o l'istituzione di centri di detenzione o di spazi giuridicamente differenziati) (Cuttitta, 2007; Menjívar, 2014; Shachar, 2007). Pratiche di internalizzazione poste in essere da un paese di 'transito' potranno anche produrre effetti in linea con gli obiettivi delle pratiche di esternalizzazione dei paesi di destinazione, ma l'internalizzazione può ben essere frutto di decisioni autonome delle autorità locali, indipendenti dalle spinte esternalizzatrici.

A questo proposito conviene peraltro ricordare che i regimi nazionali di detenzione e deportazione - per restare su questo esempio - sono ormai sempre più un miscuglio di elementi autonomi ed eteronomi, di internalizzazione dei paesi di origine o 'transito' e di esternalizzazione europea (Adam et al., 2020). Da un lato, infatti, essi possono essere il frutto di leggi criminalizzanti e di pratiche repressive consolidate che precedono l'esternalizzazione o prescindono dall'esternalizzazione; dall'altro, essi vedono la partecipazione di elementi tipici dell'esternalizzazione, come i progetti di rimpatrio volontario assistito portati avanti dall'Organizzazione Internazionale per le Migrazioni (OIM) o le attività dell'Alto Commissariato delle Nazioni Unite per i Rifugiati (ACNUR) funzionali alla creazione, in determinate regioni del mondo, di spazi di protezione idonei a giustificare le politiche che tengono i richiedenti asilo lontani dal territorio europeo. In effetti il ruolo delle organizzazioni internazionali (OI) come l'OIM o l'ACNUR nei processi di esternalizzazione è abbastanza evidente, se non altro perché queste organizzazioni vivono dei finanziamenti che ricevono dai governi di quegli stessi stati che guidano le politiche di esternalizzazione (Lavenex, 2016; Wunderlich, 2012).

\section{STATO-CENTRISMO}

Il cenno alle OI, fatto in chiusura della sezione precedente, introduce il passaggio dalla questione dell'eurocentrismo a quella dello stato-centrismo.

Gli studi sulle migrazioni e sui confini, infatti, si sono sempre più interrogati non solo sul dove e sul come del confine (dove si manifesta, in che forma, con quali modalità operative...), ma anche su chi i confini li traccia. Chi crea e trasforma i confini? Chi li aggira, li fortifica, li mette in discussione, li abbatte e li ricostruisce? Chi partecipa all'opera di bordering, de-bordering e re-bordering?

Le dinamiche relative a migrazioni internazionali e confini statali, in effetti, non sono più una questione sulla quale sono solo gli stati a pronunciarsi e ad agire. Di queste dinamiche sono corresponsabili soggetti diversi, comprese varie categorie di attori non statali: dalle OI alle ONG, dall'industria della sicurezza ai vari gruppi, più o meno organizzati, della società civile, comprese le associazioni o comunità informali di migranti, fino ai migranti stessi e alla schiera di soggetti che facilitano i loro viaggi (dai familiari agli intermediari, dai trafficanti improvvisati a quelli inseriti in grandi organizzazioni transnazionali).

Questi diversi attori hanno a loro volta natura e motivazioni differenti. Rumford (2008, p. 8), per esempio, nota che "alcuni attori della società civile lavorano per erodere i confini, mentre altri lavorano per rafforzarli o per crearne di nuovi" (trad. d. aut.). Con riferimento all'esternalizzazione, lo stesso vale anche per attori statali e OI. Tutti questi soggetti possono essere più o meno indipendenti e autonomi, e le loro motivazioni e attività possono essere più o meno in linea con i processi di esternalizzazione. Le azioni da essi poste in essere possono avere effetti irrilevanti rispetto a tali processi, oppure possono supportarli, oppure, ancora, possono agire in contrasto con essi. Che siano favorevoli o contrarie, poi, le diverse azioni possono avere un grado più o meno elevato di efficacia. Inoltre, gli attori stessi possono essere più o meno consapevoli della relazione tra le loro scelte e i processi in questione.

Molto è stato scritto, e a ragione, sul ruolo delle OI come strumenti di esternalizzazione (sull'OIM: Andrijasevic e Walters, 2010; Ashutosh e Mountz, 2011; sull'ACNUR: Valluy, 2007; sull'ICMPD: Cobarrubias, 2019). Considerarle unicamente come strumenti al servizio delle politiche di esternalizzazione dei paesi più ricchi sarebbe però riduttivo. Le OI possono esprimere una propria progettualità, influendo sulle scelte dei paesi di destinazione (Geiger, 2010; Hess, 2010), e anche svolgere una funzione di contrappeso nei confronti degli stati esternalizzanti (Lavenex, 2016). Più in generale, esse sono costantemente impegnate in un'opera di mediazione tra interessi spesso confliggenti: quelli dei paesi di destinazione, quelli dei paesi di origine e 'transito' nei quali si trovano a operare, e quelli nominalmente tutelati dai loro mandati istituzionali.

A volte, poi, le pratiche delle OI possono produrre effetti contrari agli obiettivi perseguiti dalle politiche di esternalizzazione. È il caso, per esempio, dei contributi in denaro offerti in certe circostanze dall'ACNUR ai suoi assistiti. Nel 2013 le autorità tunisine stabilirono la chiusura del campo profughi di Choucha, che in quell'area desertica aveva ospitato centinaia di migliaia di persone fuggite dalla Libia in guerra nel 2011. In tale circostanza l'ACNUR offrì ai pochi rifugiati che ancora vivevano nel campo 1500 dinari affinché accettassero il trasferimento in contesti urbani. L'agenzia dell'ONU era ben consapevole del fatto che quella somma corrispondeva alla tariffa minima di una traversata dalla Libia per l'Italia. Infatti la maggior parte delle persone che aderirono alla proposta, anziché stabilirsi in un centro abitato tunisino, attraversò il confine libico e s'imbarcò alla volta della Sicilia (Cuttitta, 
2020, p. 5; Garelli e Tazzioli, 2017, pp. 23-24). Lo stesso si può dire dell'urban package offerto dall'ACNUR, nel 2020, alle persone costrette a lasciare il centro di raccolta e partenza di Tripoli, in Libia. Il risultato di queste azioni, lungi dall'essere in linea con quelli attesi dall'esternalizzazione, sembra essere esattamente l'opposto, e se è difficile sostenere che l'obiettivo dell'ACNUR sia proprio quello di consentire ai loro assistiti di prendere il mare, è evidente come l'agenzia dell'ONU sia perfettamente consapevole che questo sarà un probabile effetto della sua azione.

Rispetto alle OI, meno è stato scritto sulle ONG. A volte le iniziative portate avanti da ONG in paesi di 'transito', nell'ambito di progetti finanziati dall'Europa (spesso tramite OI), finiscono per favorire il movimento verso l'Europa, anche se il loro obiettivo sarebbe fare restare le persone dove sono. È il caso di alcuni progetti per l'integrazione economica dei migranti - per esempio tramite azioni di micro-credito volte a sviluppare progettualità imprenditoriali - in paesi di 'transito' come la Tunisia o l'Egitto (Cuttitta, 2020). Questi progetti sono concepiti come strumenti di esternalizzazione, nella misura in cui l'integrazione economica favorirebbe quella sociale e quindi il radicamento nel territorio, e di conseguenza spingerebbe i beneficiari a rinunciare all'eventuale idea di trasferirsi in Europa. Essi, tuttavia, possono finire per produrre effetti contrari se i beneficiari usano i soldi ottenuti (a volte grazie all'attività economica avviata; altre volte già ben prima, vendendo le attrezzature loro fornite al fine di avviare tale attività) proprio per finanziare il viaggio verso l'Europa, anziché per integrarsi nella società del paese di 'transito'.

Alcune ONG in paesi di 'transito', poi, oscillano tra attività di esternalizzazione e attività di supporto alla mobilità dei migranti, oppure si dedicano ad attività catalogabili come esternalizzazione (per esempio quelle che mirano all'integrazione culturale o che offrono assistenza legale e sanitaria, o ancora, e ancor più, le campagne di informazione) cercando di interpretarle in modo critico, con l'obiettivo di consentire ai destinatari di prendere le decisioni sul loro futuro in modo autonomo e consapevole.

Se gli esempi qui proposti si limitano a OI e ONG, non va dimenticato il ruolo dei tanti altri soggetti non statali: dai gruppi di attivisti alle reti sociali dei migranti; dai vettori alle agenzie di intermediazione consolare per il rilascio dei visti; dall'industria della sicurezza a quella del traffico; etc.

\section{CONCETTI E METODI}

$\mathrm{Al}$ fine di cogliere e riflettere la molteplicità e varietà delle pulsioni e delle dinamiche tra i diversi protagonisti, nonché delle mutevoli spazialità e temporalità del confine, i migration and border studies, negli ultimi due decenni, hanno sviluppato, o riadattato e fatto propri, diversi concetti.

La nozione di borderwork (Rumford, 2006), per esempio, indica l'insieme di attività di bordering, de-bordering e rebordering non semplicemente in quanto tali ma proprio in quanto poste in essere da attori diversi. Il concetto di borderscape (Brambilla, 2015; Perera, 2007) allude a un caleidoscopico spazio geo-politico-culturale creato e caratterizzato dalle molteplici interazioni, simboliche e materiali, che ruotano attorno a un confine fisico, e che sono poste in essere da diversi attori. Similmente, il concetto di regime, nelle sue varie declinazioni (regime migratorio, regime di frontiera, etc.), consente di "esplorare i molteplici intrecci tra discorsi, rapporti di potere e soggettività" (Horvath et al., 2017, p. 305, trad. d. aut.), di guardare al confine come a una sede di conflitti e di negoziazioni tra attori, interessi, logiche, strutture e pratiche diversi, non inquadrabili in un'unica logica ordinatrice (Hess, 2012; Tsianos e Karakayal1, 2010). O ancora il concetto di assemblaggio (Nail, 2017). Derivato da Deleuze e Guattari (1980), ${ }^{2}$ tale concetto è stato ripreso dalla letteratura sulla globalizzazione (Collier e Ong, 2005; Sassen, 2006) e applicato anche ai migration and border studies, per lo più con riferimento agli assemblaggi di controllo (Allen e Vollmer, 2017; Casas-Cortés e Cobarrubias, 2019; Fisher, 2018), intesi come il risultato contingente della mutevole interazione tra diversi attori, territori e dispositivi, che si sviluppa in modo non necessariamente coerente e in assenza di un centro di coordinamento o direzione unitario. Complementare ai precedenti è infine il concetto di matrice bourdieusiana di 'campo', esemplificato dai transnational social fields di Levitt e Glick Schiller (2004) o dagli champs dei professionisti della gestione dell'insicurezza di Bigo (2005).

Analizzare gli sviluppi dei fenomeni migratori e le dinamiche dei confini da queste prospettive richiede anche approcci metodologici che siano attenti alla diversità non solo degli attori ma anche dei livelli - formali e informali, istituzionali e interpersonali - e delle scale geografiche ai quali si producono, si vivono e si trasformano i confini. Da qui gli approcci ispirati alla ethnographische Grenzregimeforschung (Hess, 2012; Hess et al., 2018), alla multi-sited ethnography (Falzon, 2009) o, ancora, alla non-local ethnography (Feldman, 2012). Quest'ultima individua nelle dinamiche relazionali sovraterritoriali - per esempio tra burocrazie e tecnocrazie pubbliche e private - una chiave per cogliere aspetti importanti di processi che invece hanno un carattere profondamente territoriale, e quindi anche locale, come quelli, appunto, relativi ai controlli delle migrazioni internazionali.

Come ricordano Üstübici, Stock e Schultz (2019), inoltre, l'esternalizzazione ha uno stretto rapporto anche con il concetto di 'management' ('migration management' o 'border management'), nella misura in cui il know-bow

2 'Agencement' nell'originale francese, 'concatenamento' nella traduzione italiana, 'assemblage' in quella inglese. 
necessario per una corretta 'gestione' dei confini e delle frontiere è parte del capitale trasferito - direttamente o per il tramite delle OI - dai paesi del 'Nord' a quelli del 'Sud' globale, e a loro volta i parametri gestionali ai quali sono chiamati a uniformarsi questi ultimi sono mirati a facilitare il controllo delle migrazioni da parte dei primi. Il paradigma della 'gestione', grazie al suo sapore manageriale, tecnocratico e depoliticizzato (Pécoud, 2015), si è andato negli ultimi vent'anni progressivamente affiancando, fin quasi a sostituirlo, a quello del controllo. E proprio con la sua aura di apoliticità esso facilita l'adattamento dei paesi di origine e 'transito' alle politiche di esternalizzazione decise e attuate dai paesi di destinazione del 'Nord globale' (Zaiotti, 2016). Si evidenzia così la correlazione tra il paradigma della gestione e l'esternalizzazione. La prospettiva del migration management, però, è meno stato-centrica di quella dell'esternalizzazione. L'approccio 'gestionale' sostiene più generalmente, come osservano Geiger e Pécoud (2013), il disciplinamento globale della mobilità. Analizzare la logica di questo disciplinamento consente di andare oltre le dinamiche inter-statali e di esaminare altre relazioni: per esempio, quella tra le frontiere del capitale e i confini territoriali (Mezzadra e Neilson, 2014; Mezzadra, 2015), che comprende i processi statali di esternalizzazione ma va anche al di là di essi.

De-nazionalizzare lo sguardo significa quindi, da un lato, dare importanza anche agli attori diversi da quelli statali, come le ONG o anche i privati cittadini impegnati in varie forme di borderwork. Dall'altro lato, denazionalizzare significa anche sottolineare che il funzionamento dei confini degli stati risponde a razionalità che travalicano logiche e interessi di carattere statale.

Il paradigma dell'esternalizzazione si basa sulla classica contrapposizione binaria tra dentro e fuori, tra interno ed esterno, che, alla luce dei più complessi processi della globalizzazione, ha certamente perso parte della propria capacità di riflettere una realtà in cui il dentro e il fuori, l'interno e l'esterno appaiono sempre più indistinguibili (Bigo, 2001; Walker, 2005). Se da un lato il termine 'esternalizzazione' sembrerebbe nostalgicamente aggrapparsi a questa contrapposizione ('esterno esplicitato dal termine implica l'esistenza di un interno), dall'altro lato il fatto stesso che le politiche migratorie di un paese, e quindi i confini di un paese, possano riemergere al di fuori del relativo territorio, addirittura nel territorio di un altro paese, costituisce un chiaro indicatore della dissoluzione dell'illusione westfaliana, del carattere convenzionale della sovranità territoriale.

A meglio comprendere questa ambiguità del termine 'esternalizzazione' può aiutare il soffermarsi sul significato di un altro termine - 'delocalizzazione' - a esso correlato ma da esso distinto. Questo termine (almeno nelle sue varianti inglese e francese, delocalisation/delocalization/délocalisation) è infatti impiegato sia come semplice sinonimo di esternalizzazione (Albahari, 2015; Nieuwenhuys e Pécoud, 2007; Oliveira Martins e Strange, 2019; Ryan e Mitsilegas, 2010; Valluy, 2005), sia in senso più ampio, come termine-contenitore, a indicare l'insieme delle diverse modalità (esternalizzate e internalizzate) attraverso le quali i confini statali si manifestano e assolvono alle proprie funzioni lontano dal tracciato delle linee di demarcazione ufficiali (Bennafla, 2002; Bigo e Guild, 2003; Cuttitta, 2018; Salter, 2004). In quest'ultima accezione, la parola 'delocalizzazione' ha il vantaggio - rispetto a 'esternalizzazione' e 'internalizzazione' - della neutralità direzionale, dal momento che non assume una prospettiva unilaterale che implichi una dinamica spaziale netta (dall'esterno verso l'interno o viceversa). Inoltre, il termine lascia aperta, e consente di affrontare meglio, la questione precedentemente introdotta: 'chi traccia il confine?' (Rumford, 2006). Ciò appare particolarmente opportuno in un contesto in cui la moltiplicazione degli attori - sia statali, sia non statali - rende sempre più difficile individuare i diversi soggetti coinvolti nelle attività di bordering, debordering e re-bordering, e stabilire in che misura ciascuno di essi contribuisca a determinare le dinamiche alla base delle trasformazioni dei confini.

Più in generale, ciò appare in linea con le diverse idee di governance o governamentalità diffusa, di "un mondofrontiera nel quale i confini sono ovunque, molteplici sono le loro identità, mutevoli le forme sotto le quali essi si manifestano, diverse le modalità con le quali essi operano" (Cuttitta, 2007, p. 156), un mondo in cui le riconfigurazioni dei confini statali - come dei confini territoriali di ogni genere - sono sempre più spesso riconducibili a contestuali riconfigurazioni di dinamiche economiche globali; in cui le modalità con cui i controlli delle migrazioni e dei confini sono regolamentati e posti in essere sembrano sempre più espressioni di un apparato anonimo e 'acefalo' (Feldman, 2012).

\section{CONCLUSIONI}

Questo articolo si è concentrato sul tema dell'esternalizzazione dei controlli migratori (assumendo che quanto osservato a tal proposito sia riferibile anche agli studi su migrazioni e confini in generale) per riflettere criticamente su come stato-centrismo e nord-centrismo - intesi come la tendenza ad assumere, rispettivamente, lo stato e il 'Nord globale' come riferimenti assoluti della ricerca sociale - possano determinare distorsioni prospettiche e limitare il campo visivo del ricercatore. In particolare, a soffrire di tali distorsioni e limitazioni può essere lo studio delle dinamiche migratorie e frontaliere nelle regioni e nei paesi convenzionalmente classificati come di origine e/o di 'transito'. 
Il rischio di nord-centrismo, in questi casi, consiste nel lasciarsi risucchiare dalla prospettiva univoca dell'esternalizzazione, interpretando le migrazioni, i confini e i relativi controlli - cioè i fenomeni oggetto di studio - soltanto come migrazioni verso il 'Nord globale', come confini del 'Nord globale', come controlli voluti dai paesi del 'Nord globale' (e quindi funzionali agli interessi di questi ultimi), e i paesi di origine e 'transito' soltanto come soggetti passivi, costretti a seguire ciecamente le direttive di quelli esternalizzanti. L'articolo ha invece sottolineato l'esigenza di considerare anche l'agency degli 'esternalizzandi'. Questa può manifestarsi, in primo luogo, nella contrapposizione all'esternalizzazione o nell'uso del potere contrattuale che le richieste di esternalizzazione inevitabilmente conferiscono a chi le riceve. In secondo luogo, l'agency degli 'esternalizzandi' può esplicitarsi tramite determinate politiche e pratiche in materia di migrazioni e confini, le quali indipendentemente dal fatto che esse siano più o meno in linea con gli obiettivi degli 'esternalizzanti', o non lo siano per niente - possono essere poste in essere dagli 'esternalizzandi' in modo autonomo, rispondendo a esigenze di carattere nazionale o regionale piuttosto che a sollecitazioni ricevute, nel segno dell'esternalizzazione, dai più influenti paesi di destinazione.

Non si tratta certamente di negare né la cornice di subordinazione e asimmetria che caratterizza le relazioni internazionali tra paesi ricchi e paesi poveri, tra paesi del 'Nord' e del 'Sud' globali, tra paesi di destinazione e paesi di origine o 'transito', né la matrice neo-coloniale di tale contesto. Si tratta piuttosto di evitare che la prospettiva del ricercatore si appiattisca nell'ego-centrismo e nel concentricismo che contraddistinguono l'oggetto del suo studio (l'esternalizzazione), e di favorire una visione a più ampio spettro, che tenga conto di diverse variabili, a cominciare dai diversi attori in campo e dai rispettivi interessi.

Proprio la questione degli attori in campo è alla base della critica all'altro -ismo preso in considerazione da questo articolo: lo stato-centrismo. L'esternalizzazione è, chiaramente, un fenomeno che vede primi protagonisti gli stati. Fermarsi alle relazioni tra stati, tuttavia, impedisce di cogliere la complessità di un quadro generale che vede coinvolti diversi attori non statali, tra i quali l'articolo si è soffermato in particolare su OI e ONG. I diversi attori non statali, inoltre, andrebbero visti non esclusivamente nel ruolo - che tanti, peraltro, effettivamente rivestono - di strumenti dell'esternalizzazione statale ma, più in generale, come elementi che contribuiscono a determinare in vario modo le spinte esternalizzanti, promuovendole, ispirandole e orientandole secondo i propri interessi (si pensi all'industria della sicurezza), agendo come contrappeso (è il caso, per esempio, di certe OI) oppure ostacolandole, più o meno consapevolmente e volontariamente (come, tra gli altri, alcune ONG).

In conclusione, l'idea qui sviluppata è che, anche se si assume una prospettiva inevitabilmente statoterritoriale e linearmente univoca, come quando si osserva il fenomeno dell'esternalizzazione in paesi o regioni di 'transito', questa possa essere compensata e relativizzata, riducendone la carica stato-centrica e nord-centrica. L'obiettivo può essere perseguito attraverso uno sguardo più multi-prospettico (Rumford, 2012) e caleidoscopico (Brambilla, 2015), che provi ad aprirsi all'azione dei diversi attori in campo e a sganciarsi dalla fissa, unidirezionale linearità dell'asse centro-margini. A tal fine può risultare utile il ricorso ad alcuni concetti (borderwork, borderscape, regime, assemblaggio, campo, delocalizzazione) e approcci metodologici (ethnographische Grensregimeforschung, multi-sited ethnography, non-local ethnography) passati in rassegna nell'ultima sezione dell'articolo. L'articolo ha insistito in particolare sul concetto di 'delocalizzazione'. Partendo da un altro aspetto critico del concetto di esternalizzazione - il rapporto tra interno ed esterno - si è ipotizzato che il concetto di 'delocalizzazione' possa rivelarsi utile in virtù della sua neutralità direzionale. Questa, relativizzando il rapporto con lo spazio, consente al concetto di 'delocalizzazione' di inserirsi coerentemente nel contesto di un 'mondofrontiera' in cui i confini, parallelamente alla loro proliferazione, vedono le proprie identità e finalità confondersi, mentre le loro categorie fondative - il 'dentro' e il 'fuori' - diventano sempre più indistinguibili.

RIFERIMENTI BIBLIOGRAFICI

Adam I., Trauner F., Jegen L. e Roos C. (2020) West African interests in (EU) migration policy. Balancing domestic priorities with external incentives. Journal of Ethnic and Migration Studies. DOI: 10.1080/1369183X.2020.1750354

Agnew J. (1994) The Territorial Trap: The Geographical Assumptions of International Relations Theory. Review of International Political Economy 1(1), 53-80. DOI: 10.1080/09692299408434268

Albahari M. (2015) Crimes of Peace. Mediterranean Migrations at the World's Deadliest Border. Philadelphia: University of Pennsylvania Press.

Allen W.L. e Vollmer B.A. (2017) Clean skins: Making the e-Border security assemblage. Environment and Planning D: Society and Space 36(1), 23-39. DOI: $10.1177 / 0263775817722565$

Andreas P. (2003) Redrawing the Line. Borders and Security in the Twenty-first Century. International Security 28(2), 78-111. DOI: $10.1162 / 016228803322761973$

Andrijasevic R. e Walters W. (2010) The International Organization for Migration and the international government of borders. Environment and Planning D: Society and Space 28(6), 977-999. DOI: 10.1068/d1509

Ashutosh I. e Mountz A. (2011) Managing migration for the benefit of whom? Interrogating the work of the International Organization for Migration. Citizenship Studies 15(1), 21-38. DOI: 10.1080/13621025.2011.534914

Bartels I. (2018) Practices and power of knowledge dissemination. International organizations in the externalization of migration management in Morocco and Tunisia. Movements. Journal für kritische Migrations- und Grenzregimeforschung 4(1), 47-66, testo disponibile al 
sito: https://movements-journal.org/issues/06.wissen/03.bartels--practices-and-power-of-knowledge-dissemination-internationalorganizations-in-the-externalization-of-migration-management-in-morocco-and-tunisia.pdf (consultato il 29 aprile 2020).

Beaumont P. e Smith H. (2019) Erdoğan: I'll let Syrian refugees leave Turkey for west unless safe zone set up, The Guardian, 5 settembre, testo disponibile al sito: https://www.theguardian.com/world/2019/sep/05/erdogan-ill-let-syrian-refugees-leave-turkey-for-westunless-safe-zone-set-up (consultato il 19 aprile 2020).

Bennafla K. (2002) Les frontières africaines : nouvelles significations, nouveaux enjeux. Bulletin de l'Association de géographes français, 79 (2), 134146. DOI: $10.3406 /$ bagf.2002.2265

Bialasiewicz L. (2012) Off-shoring and out-sourcing the borders of EUrope: Libya and EU border work in the Mediterranean. Geopolitics 17(4), 843-866. DOI: 10.1080/14650045.2012.660579

Bigo D. (2005) La mondialisation de l'(in)sécurité ? Cultures \& Conflits 58, 53-101. DOI: 10.4000/conflits.1813

Id. (2001) The Möbius Ribbon of Internal and External security. In: Albert M., Jacobson D. e Lapid Y., a cura di, Identity, borders, orders: Retbinking International Relations Theory, Minneapolis: University of Minnesota Press.

Id. e Guild E. (2003) Le visa Schengen: expression d'une stratégie de "police" à distance. Cultures \& Conflits 49, 22-37. DOI: $10.4000 /$ conflits. 924

Bisong A. (2019) Trans-regional institutional cooperation as multilevel governance: ECOWAS migration policy and the EU. Journal of Ethnic and Migration Studies 45(8), 1294-1309. DOI: 10.1080/1369183X.2018.1441607

Brachet J. (2018) Manufacturing Smugglers: From Irregular to Clandestine Mobility in the Sahara. Annals of the American Academy of Political and Social Science 676(1), 16-35. DOI: 10.1177/0002716217744529

Brambilla C. (2015) Exploring the critical potential of the borderscapes concept. Geopolitics 20(1), 14-34. DOI: 10.1080/14650045.2014.884561

Bredeloup S. e Pliez O. (2005) Migrations entre les deux rives du Sahara. Autrepart 36, 3-20. DOI: 10.3917/autr.036.0003

Carrera S., den Hertog L., Panizzon M. e Kostakopoulou D. (2019), a cura di, EU external migration policies in an era of global mobilities: Intersecting policy universes. Leiden: Brill.

Casas-Cortés M. e Cobarrubias S. (2019) Genealogies of contention in concentric circles: Remote migration control and its Eurocentric geographical imaginaries. In Mitchell K., Jones R. e Fluri J., a cura di, Handbook on critical geographies of migration. Cheltenham: Edward Elgar. DOI: $10.4337 / 9781786436030.00024$

Cassarino J.-P. (2018) Beyond the criminalisation of migration: a non-western perspective. International Journal of Migration and Border Studies 4(4), 397-411. DOI: 10.1504/ijmbs.2018.096756

Id. (2005) Migration and border management in the Euro-Mediterranean area: Heading towards new forms of interconnectedness. In: IEMed (Ed.), Mediterranean Yearbook 2005 (227-231). Barcelona: IEMed.

Celata F. e Coletti R. (2016) Beyond Fortress Europe. Unbounding European normative power and the neighbourhood policy. Geography Compass 10(1), 15-24. DOI: $10.1111 /$ gec 3.12254

Cobarrubias S. (2019) Mapping illegality: The i-map and the cartopolitics of "migration management" at a distance. Antipode. DOI: $10.1111 /$ anti.12512

Id., Casas-Cortés M., Garelli G., Heller C., Pezzani L., Pickles J. e Tazzioli M. (2015) Externalization. Cultural Studies 29(1), 73-77. DOI: 10.1080/09502386.2014.891630

Collier S.J. e Ong A. (2005), a cura di, Global Assemblages: Technology, Politics and Ethics as Anthropological Problems. Oxford: Blackwell.

Collyer M. (2016) Geopolitics as a migration governance strategy: European Union bilateral relations with Southern Mediterranean countries. Journal of Ethnic and Migration Studies 42(4), 606-624. DOI: 10.1080/1369183x.2015.1106111

Cuttitta P. (2020) Non-governmental/civil society organizations and the EU-externalization of migration management in Tunisia and Egypt. Population, Space and Place. DOI: 10.1002/psp.2329

Cuttitta P. (2018) Delocalization, humanitarianism and human rights: The Mediterranean border between exclusion and inclusion. Antipode 50(3), 783-803. DOI: 10.1111/anti.12337

Cuttitta P. (2007) Segnali di confine. Il controllo dellimmigrazione nel mondo-frontiera. Milano: Mimesis

Deleuze G. e Guattari F. (1980) Mille piani. Roma: Castelvecchi.

Denaro C. (2016) The reconfiguration of Mediterranean migration routes after the war in Syria: narratives of the 'Egyptian route' to Italy (and beyond). In: Ribas Mateos N., a cura di, Migration, mobilities and the Arab Spring. Spaces of refugee flight in the Eastern Mediterranean. Cheltenham: Edward Elgar.

Düvell, F. (2012) Transit migration: A blurred and politicised concept. Population, Space and Place 18, 415-427. DOI: 10.1002/psp.631

Falzon M. (2009) Multi-Sited Ethnography: Theory, Praxis and Locality in Contemporary Research. Aldershot: Ashgate.

Feldman G. (2012) The Migration Apparatus. Security, Labor, and Policymaking in the European Union. Stanford: Stanford University Press.

Fisher D.X.O. (2018) Situating Border Control: Unpacking Spain’s SIVE border surveillance assemblage. Political Geography 65, 67-76. DOI: 10.1016/j.polgeo.2018.04.005

Gaibazzi P., Bellagamba A. e Dünnwald S. (2017), a cura di, EurAfrican borders and migration management. Political cultures, contested spaces, and ordinary lives. Basingstoke: Palgrave.

Garelli G. e Tazzioli M. (2017) Tunisia as a revolutionized space of migration. New York: Palgrave.

Eadd. (2013) Challenging the discipline of migration: militant research in migration studies, an introduction. Postcolonial Studies 16(3), $245-$ 249. DOI: $10.1080 / 13688790.2013 .850041$

Geiger M. (2010) Mobility, development, protection, EU-integration! The IOM’s national migration strategy for Albania. In: Geiger M. e Pécoud A., a cura di, The politics of international migration management. Basingstoke: Palgrave.

Id. e Pécoud A. (2013), a cura di, Disciplining the transnational mobility of people. Basingstoke: Palgrave.

Genç F., Heck G. e Hess S. (2018). The multilayered migration regime in Turkey: Contested regionalization, deceleration and legal precarization. Journal of Borderlands Studies. DOI: 10.1080/08865655.2017.1344562

Hess S. (2012) De-naturalising transit migration. Theory and methods of an ethnographic regime analysis. Population, Space and Place 18(4), 428-40. DOI: $10.1002 /$ psp.632

Ead. (2010) 'We are facilitating states!' An ethnographic analysis of the ICMPD. In: Geiger M. e Pécoud A., a cura di, The Politics of international migration management. Basingstoke: Palgrave. 
Ead., Kasparek B. e Schwertl M. (2018) Regime ist nicht Regime ist nicht Regime. Zum theoriepolitischen Einsatz der ethnografischen (Grenz-)Regimeanalyse. In: Pott A., Rass C. e Wolff F., a cura di, Was ist ein Migrationsregime? What Is a Migration Regime? Wiesbaden: Springer VS.

Horvath K., Amelina A. e Peters K. (2017) Re-thinking the politics of migration. On the uses and challenges of regime perspectives for migration research. Migration Studies 5(3), 301-314. DOI: 10.1093/migration/mnx055

Isşleyen B. (2018) Transit mobility governance in Turkey. Political Geography 62, 23-32. DOI: 10.1016/j.polgeo.2017.09.017

Kramsch O.T. (2011) Along the Borgesian frontier: Excavating the neighbourhood of "Wider Europe". Geopolitics 16(1), 193-210. DOI: $10.1080 / 14650045.2010 .493786$

Lahav G. e Guiraudon V. (2000) Comparative perspectives on border control: Away from the border and outside the state. In: Andreas P. e Snyder T., a cura di, The Wall Around the West: State Borders and Immigration Controls in North America and Europe. Lanham: Rowman \& Littlefield.

Lavenex S. (2016) Multilevelling EU external governance: the role of international organizations in the diffusion of EU migration policies. Journal of Ethnic and Migration Studies 42(4), 554-570. DOI: 10.1080/1369183x.2015.1102047

Levitt P. e Glick Schiller N. (2004) Conceptualizing Simultaneity: A Transnational Social Field Perspective on Society. International Migration Review 38(3), 1002-1039. DOI: 10.1111/j.1747-7379.2004.tb00227.x

Menjívar C. (2014) Immigration law beyond borders: Externalizing and internalizing border controls in an era of securitization. Annual Review of Law and Social Science 10, 353-369. DOI: 10.1146/annurev-lawsocsci-110413-030842

Mezzadra S. (2015) Confini, frontiere, capitale. Intrasformazione. Rivista di storia delle idee 4(2), 20-24.

Id. e Neilson B. (2014) Confini e frontiere. La moltiplicarione del lavoro nel mondo globale. Bologna: Il Mulino.

Nail T. (2017) What is an assemblage? SubStance 46(1), 21-37. DOI: 10.3368/ss.46.1.21

Nieuwenhuys C. e Pécoud A. (2007) Human trafficking, information campaigns, and strategies of migration control. American Bebavioral Scientist 50(12), 1674-1695. DOI: 10.1177/0002764207302474

Okunade S.K. e Ogunnubi O. (2018) A "Schengen" Agreement in Africa? African Agency and the ECOWAS Protocol on Free Movement. Journal of Borderlands Studies. DOI: 10.1080/08865655.2018.1530128

Oliveira Martins B. e Strange M. (2019) Rethinking EU external migration policy: contestation and critique. Global Affairs, 5:3, $195-202$. DOI: $10.1080 / 23340460.2019 .1641128$

Paoletti E. (2010) The migration of power and North-South inequalities. The case of Italy and Libya. Basingstoke: Palgrave.

Parker N. (2008) A theoretical introduction: spaces, centers, and margins. In: Parker N., a cura di, The geopolitics of Europe's identity. Centers, boundaries, and margins. Basingstoke: Palgrave.

Pécoud A. (2015) Depoliticising migration. Global governance and international migration narratives. Basingstoke: Palgrave.

Perera S. (2007) A Pacific zone? (In)Security, sovereignty, and stories of the Pacific borderscape. In: Rajaram P.-K. e Grundy-Warr C., a cura di, Borderscapes. Hidden geographies and politics at territory's edge. Minneapolis: Minnesota University Press.

Pliez O. (2006) La frontiera tra la Libia e il Sahel. Uno spazio migratorio rimesso in discussione. In: Cuttitta P. e Vassallo Paleologo F., a cura di, Migrazioni, frontiere, diritti. Napoli: Edizioni scientifiche italiane.

Id. (2004) De l'immigration au transit ? La Libye dans l'espace migratoire euro-africain. in Pliez O., a cura di, La nowvelle Libye. Societés, espaces et géopolitique au lendemain de l'embargo. Parigi: Karthala.

Poulantzas N. (1978) L'Etat, le pouvoir, le socialisme. Paris: PUF.

Raffestin C. (1981) Per una geografia del potere. Milano: Unicopli.

Reslow N. (2019) EU External migration policy: taking stock and looking forward. Global Affairs 5(3), 273-278. DOI: $10.1080 / 23340460.2019 .1604071$

Ead. (2012) The role of third countries in EU migration policy: The Mobility Partnerships. European Journal of Migration and Law 14(4), 393415. DOI: $10.1163 / 15718166-12342015$

Rumford C. (2012) Towards a multiperspectival study of borders. Geopolitics 17(4), 887-902. DOI: 10.1080/14650045.2012.660584

Id. (2008) Introduction: Citizens and borderwork in Europe. Space and Polity 12(1), 1-12. DOI: 10.1080/13562570801969333

Id. (2006) Theorizing borders. European Journal of Social Theory 9(2), 155-169. DOI: 10.1177/1368431006063330

Ryan B. e Mitsilegas V. (2010), a cura di, Extraterritorial Immigration Control: Legal Challenges. Leiden-Boston: Brill.

Salter, M.B. (2004) Passports, mobility, and security: How smart can the border be? International Studies Perspectives 5(1), 71-91.

Sassen S. (2006) Territory, Authority, Rights. From Medieval to Global Assemblages. Princeton/Oxford: Princeton University Press.

Sciortino G. (2000) L'ambizione della frontiera. Le politiche di controllo migratorio in Europa. Milano: Franco Angeli.

Shachar A. (2007) The Shifting Border of Immigration Regulation. Stanford Journal of Civil Rights and Civil Liberties 3(2), 165-193.

Stock I. (2019) Time, Migration and Forced Immobility. Sub-Sabaran African Migrants in Morocco. Bristol: Bristol University Press.

Trauner F. e Deimel S. (2013) The Impact of EU Migration Policies on African Countries: the Case of Mali. International Migration 51(4), 2032. DOI: 10.1111 /imig.12081

Tsianos V. e Karakayalı S. (2010) Transnational Migration and the Emergence of the European Border Regime: An Ethnographic Analysis. European Journal of Social Theory 13(3), 373-387. DOI: https://doi.org/10.1177/1368431010371761

Üstübici A., Stock I. e Schultz S. (2019) Externalization at work: responses to migration policies from the Global South. Comparative Migration Studies. DOI: 10.1186/s40878-019-0157-z

Valluy J. (2007) Contribution à une sociologie politique du HCR: le cas des politiques européennes et du HCR au Maroc. TERRA-HN. Recueil Alexandries. Collection 'Etudes'1, testo disponibile al sito: https://reseau-terra.eu/article571.html (consultato il 28 aprile 2020).

Id. (2005) La nouvelle Europe politique des camps d'exilés: genèse d'une source élitaire de phobie et de répression des étrangers. Cultures \& Conflits 57, 13-69. DOI: $10.4000 /$ conflits.1726

van Houtum H. e Boedeltje F. (2011) Questioning the EU's neighbourhood geo-politics: Introduction to a special section. Geopolitics 16(1), 121-129. DOI: $10.1080 / 14650045.2010 .493779$

Walker R.B.J. (2005) L'enigma dell'internazionale. Conflitti Globali 1(2), 42-57.

Walters W. (2015) Reflections on Migration and Governmentality. Movements. Journal für kritische Migrations- und Grenæregimeforschung 1(1), testo disponibile al sito: http://movements-journal.org/issues/01.grenzregime/04.walters--migration.governmentality.html (consultato il 29 aprile 2020).

Wimmer A. e Glick Schiller N. (2002) Methodological Nationalism and Beyond: Nation-State Building, Migration and the Social Sciences. Global Networks 2(4), 301-334. DOI: 10.1111/1471-0374.00043 
Wunderlich D. (2012) Europeanization through the grapevine: Communication gaps and the role of international organizations in implementation networks of EU external migration policy. European Integration 34(5), 485-503. DOI: 10.1080/07036337.2011.611385

Zaiotti R. (2016), a cura di, Externalizing migration management. Europe, North America and the spread of 'remote control'practices. Abingdon: Routledge. Zolberg A. (2003) The archaeology of remote control. In: Fahrmeir A., Faron O. e Weil P., a cura di, Migration control in the North Atlantic world: The evolution of state practices in Europe and the United States from the French Revolution to the Inter-War Period. New York: Berghahn. 